\title{
SAMW und Bangerter-Stiftung lancieren Förderprogramm
}

\section{Myriam Tapernoux}

Dr., Leiterin Ressort Wissenschaft, SAMW, Bern

\begin{abstract}
Klinisch forschende Ärztinnen und Ärzte sehen sich heute mit vielfältigen Herausforderungen konfrontiert. Darunter die mangelnde Zeit, insbesondere während der ärztlichen Weiterbildung, sowie die raren finanziellen Mittel, die zur Durchführung von klinischen Forschungsprojekten zur Verfügung stehen. Ein neues Förderprogramm der SAMW und der Bangerter-Stiftung soll hier Verbesserung bringen.
\end{abstract}

Die Frage, ob junge Forschende fachliche und ideelle Unterstützung erhalten, hängt häufig davon ab, wie gross das entsprechende Engagement und auch die Qualifikation ihrer Vorgesetzten ist, d.h., eine systemimmanente Unterstützung ist derzeit nicht gegeben. Das Rüstzeug für die klinische Forschung, etwa das Erwerben von theoretischen und praktischen Grundlagen, müssen sich junge Forschende weitgehend selbst organisieren. Zum anderen müssen sie Lohneinbussen in Kauf nehmen, weil ein Engagement in der Forschung immer noch weitaus geringer entlöhnt wird als die Arbeit in der Klinik.

\section{Das Programm fördert den Einstieg von jungen Medizinerinnen und Medizinern in die klinische Forschung.}

Mit dem Förderprogramm «Nachwuchs in klinischer Forschung» möchten die Gottfried und Julia BangerterRhyner-Stiftung und die Schweizerische Akademie der Medizinischen Wissenschaften (SAMW) dazu beitragen, dass mehr junge Medizinerinnen und Mediziner den Einstieg in die klinische Forschung wagen. Gut ausgebildeter wissenschaftlicher Nachwuchs ist die Voraussetzung, um die Qualität der klinischen Forschung in der Schweiz zu steigern und langfristig zu sichern. Hier setzt dieses Förderprogramm an und trägt damit auch der «Roadmap zur Nachwuchsförderung in der klinischen Forschung» Rechnung, die 2016 vom Bundesamt für Gesundheit veröffentlicht wurde (siehe Kasten).

\section{Ziele des Förderprogramms}

Die Bangerter-Stiftung und die SAMW haben am 21. März 2017 eine Vereinbarung betreffend die Durch- führung des Förderprogramms unterzeichnet. Diese vorerst auf die Jahre 2017-2020 beschränkte Initiative hat zum Ziel,

- den Einstieg von jungen Medizinerinnen und Medizinern in die klinische Forschung zu fördern;

- die Qualität der klinischen Forschung zu steigern, damit die Schweiz einen Spitzenplatz in Forschung und Innovation hält und stärkt.

\section{Roadmap zur Nachwuchsförderung in der klinischen Forschung}

Ein im Rahmen des Masterplans des Bundes zur Stärkung der Biomedizinischen Forschung und Technologie erarbeiteter Bericht kam 2014 zum Schluss, dass bei der Nachwuchsförderung in der klinischen Forschung bislang zu wenig unternommen wurde. Daraufhin übersetzte eine vom BAG eingesetzte TaskForce die Empfehlungen des Berichtes in konkrete Massnahmen, legte die entsprechenden Zuständigkeiten fest und definierte einen Zeitplan für eine erfolgreiche und nachhaltige Umsetzung der Empfehlungen.

Diese «Roadmap 2016-2021 zur Nachwuchsförderung in der klinischen Forschung" umfasst insgesamt fünf Arbeitspakete. Sie soll dazu beitragen, die bestehenden Lücken im Fördertrack für klinische Forscherinnen und Forscher zu schliessen und ihnen hochqualifizierte und effizienteTrainingsmöglichkeiten zur Verfügung zu stellen.

Um Doppelspurigkeiten zu vermeiden und die Effizienz zu erhöhen, bauen die geplanten Aktivitäten auf die bestehenden Infrastrukturen und das verfügbare Expertenwissen im Bereich der klinischen Forschung auf. Die Verantwortung für die Umsetzung der Roadmap teilen sich die lokalen MD-PhD Graduate Schools, die SCTO mit dem Netzwerk der Clinical Trial Units, die SAMW, das BAG sowie unimedsuisse.

Die geplanten Massnahmen sollen im Zeitraum von 2016 bis 2018 vorbereitet und realisiert werden, so dass ab dem Herbstsemester 2018 eine umfassende Rahmenstruktur für die Ausbildung und Unterstützung im Bereich der klinischen Forschung bereitsteht. Nach der dreijährigen Pilotphase, die eng begleitet werden soll, ist eine Evaluation vorgesehen. 
Für die Periode von 2017 bis 2020 stellt die BangerterStiftung zu diesem Zweck eine Million Franken pro Jahr zur Verfügung.

\section{Förderinstrumente und -kriterien}

Das Programm «Nachwuchs in klinischer Forschung» sieht eine zweistufige Förderung vor:

- Personenbezogene Unterstützung ermöglicht "protected research time» für Einsteiger/-innen in der klinischen Forschung (max. 75000 CHF pro Person, verteilt über ein oder zwei Jahre, davon $2 / 3$ als Stipendium und $1 / 3$ als Darlehen).

- Erfolgreiche Stipendiatinnen und Stipendiaten können anschliessend einen Beitrag zur Durchführung eines eigenen, kleinen Forschungsprojekts beantragen (max. 40000 CHF pro Jahr für max. zwei Jahre).

\section{Das Programm sieht eine zweistufige Förde- rung vor: "protected research time» für Einsteiger/-innen, gefolgt von Projektbeiträgen.}

Korrespondenz:

Dr. Myriam Tapernoux Leiterin Ressort

Wissenschaft

SAMW

Haus der Akademien

CH-3001 Bern

m.tapernoux[at]samw.ch
Zur Gesuchstellung berechtigt sind in der Schweiz tätige Ärztinnen und Ärzte, die beim Eingabetermin folgende Voraussetzungen erfüllen:

- Für ein Anfänger-Stipendium: Sie haben das Staatsexamen vor max. fünf Jahren bestanden.

- Für einen Projektbeitrag: Sie wurden durch ein Anfänger-Stipendium von diesem Programm unter- stützt und haben vor max. acht Jahren das Staatsexamen bestanden.

Ein in klinischer Forschung erfahrener Mentor garantiert eine wissenschaftliche und persönliche Unterstützung der Kandidatin/ des Kandidaten während der ganzen Dauer des Stipendiums oder des Projekts.

\section{Der erste Eingabetermin für dieses Förder- programm ist der 30. Juni 2017.}

Stipendiatinnen und Stipendiaten sind angehalten, während der unterstützten Periode Grundkenntnisse in der klinischen Forschung zu erwerben. Dazu können sie Kurse besuchen, die vom CTU-Netzwerk angeboten werden und ihrer klinischen spezialisierung und ihren Karrierezielen entsprechen.

Die Begutachtung der Anträge erfolgt durch eine Expertenkommission der SAMW. Sie entscheidet, welche der eingereichten Gesuche dem Stiftungsrat der Bangerter-Stiftung zur Förderung vorgeschlagen werden. Der erste Eingabetermin ist der 30. Juni 2017. Umfassende Informationen zur Gesuchseingabe sind online zu finden: samw.ch/nachwuchs-klinische-forschung

Informationen zu allen laufenden Förderprogrammen der SAMW unter: samw.ch/foerderung 